\title{
SMR
}

\section{Expression of transforming growth factor- $\beta 1$ and connective tissue growth factor in congenital biliary atresia and neonatal hepatitis liver tissue}

\author{
F.B. Li ${ }^{1}$, H. Zhao', K.R. Peng ${ }^{1}$, Z.G. Gao' ${ }^{2}$, S.J. Huang' ${ }^{2}$, J.F. Tou ${ }^{2}$, X.L. Shu ${ }^{3}$ \\ and W.Z. Gu ${ }^{4}$ \\ 'Department of Gastroenterology, Children's Hospital of Zhejiang University, \\ Hangzhou, China \\ 'Department of General Surgery, Children's Hospital of Zhejiang University, \\ Hangzhou, China \\ ${ }^{3}$ Laboratory of Gastroenterology, Children's Hospital of Zhejiang University, \\ Hangzhou, China \\ ${ }^{4}$ Department of Pathology, Children's Hospital of Zhejiang University, Hangzhou, \\ China \\ Corresponding author: F.B. Li \\ E-mail: fubangli669@163.com \\ Genet. Mol. Res. 15 (1): gmr.15017217 \\ Received August 15, 2015 \\ Accepted October 19, 2015 \\ Published February 19, 2016 \\ DOI http://dx.doi.org/10.4238/ gmr.15017217
}

\begin{abstract}
We investigated the expression of transforming growth factor- $\beta 1$ (TGF- $\beta 1$ ) and connective tissue growth factor (CTGF) in the liver tissue of infants with congenital biliary atresia and neonatal hepatitis, as well as the relationship between the expression of the two factors and liver fibrosis. Thirty-six infants who met the cholestasis criteria were classified into congenital biliary atresia and neonatal hepatitis groups. All specimens were stained with hematoxylin and eosin and Masson's trichrome, and the degree of liver fibrosis was assessed. The scope and level of CTGF and TGF- $\beta 1$ expression in the different specimens was evaluated by
\end{abstract}


immunohistochemistry and observation. Liver fibrosis in the congenital biliary atresia group was more advanced than that in the neonatal hepatitis group, and the difference was significant $(P<0.01)$. In the neonatal hepatitis patients, CTGF and TGF- $\beta 1$ were mainly expressed in the hepatocytes, while they were expressed in both hepatocytes and biliary epithelial cells in the congenital biliary atresia patients, and in these patients the expression was significantly stronger than in the neonatal hepatitis patients $(P<0.01)$. With the aggravation of hepatic fibrosis, CTGF and TGF- $\beta 1$ expression levels in liver tissue gradually increased, and their expression levels were significantly correlated $(P<0.01)$. Liver fibrosis is present in both congenital biliary atresia and neonatal hepatitis patients. The gradual increase of CTGF and TGF- $\beta 1$ expression levels in liver tissue is associated with liver fibrosis. Early expression of CTGF and TGF- $\beta 1$ in biliary epithelial cells may be involved in the pathogenesis of congenital biliary atresia.

Key words: Transforming growth factor- $\beta 1$; Connective tissue growth factor; Neonatal hepatitis; Congenital biliary atresia; Liver fibrosis

\section{INTRODUCTION}

Congenital biliary atresia and neonatal hepatitis are major causes of infant cholestatic jaundice. The early clinical manifestations of these two conditions are similar but the prognoses associated with them are very different. Congenital biliary atresia patients can undergo Kasai surgery to temporarily improve cholestasis. However, most patients ultimately experience cirrhosis and portal hypertension, which require liver transplantation (Kasai, 1974; Nio et al., 2003; Lee et al., 2004; Lykavieris et al., 2005). Neonatal hepatitis can be improved by symptomatic treatment, with a good prognosis. Some experts believe that the main cause of adverse prognosis in congenital biliary atresia patients is progressive liver fibrosis. Blocking or even reversing liver fibrosis may improve the prognosis. The role of liver fibrosis in neonatal hepatitis patients is still unclear. Hepatic fibrosis is an extremely complex physiological and pathological process, and the role of cytokines in the disorder elicits a great deal of interest. Transforming growth factor- $\beta 1$ (TGF- $\beta 1$ ) and connective tissue growth factor (CTGF) play key roles in liver fibrosis (Grotendorst et al., 2000; Kurikawa et al., 2003) and have therefore attracted widespread attention.

\section{MATERIAL AND METHODS}

\section{Subjects}

Thirty-six patients (21 males and 15 females) from the Department of Gastroenterology visited our hospital between September 2004 and August 2007. The youngest was 33 days old, and the oldest was 90 days old, the average age being $57.89 \pm 15.11$ days. Each baby met the cholestasis diagnostic criteria: light stool color and increased direct bilirubin accounting for more than $30 \%$ of total bilirubin. All patients had pathological liver signs (including liver enlargement and texture change) and increasing levels of alanine aminotransferase. 


\section{Samples}

All 36 patients were hospitalized and their symptoms had not improved 1-2 weeks after treatment. Liver biopsy was not contraindicated in any patient. Diagnosis was by B-mode ultrasound-guided liver tissue biopsy. Two pieces of liver tissue (1 or $2 \mathrm{~cm}$ long with more than 6 lobes, as observed under a microscope) were collected. In each case, the biopsy sample was fixed for $24 \mathrm{~h}$ in a neutral formalin solution and preserved with paraffin, followed by 1-line hematoxylin and eosin (HE) staining and Masson's trichrome staining for pathological characterization. The other sample was used for the detection of TGF- $\beta 1$ and CTGF. In two patients, normal liver tissue next to the hepatoblastoma was taken and used as a control. Both patients were males; one was 2 and the other was 4 years old. All samples were obtained with the families' consent.

\section{Sample classification and grouping}

Two experienced pathologists blinded to all specimens classified hepatic fibrosis using conventional HE and Masson's trichrome staining (referring to the 2000 Xi'an standard). The patients were classified into two groups (the neonatal hepatitis and the congenital biliary atresia group) according to the results of clinical pathology and prognosis. The inclusion criteria for the neonatal hepatitis group were: biopsy results that indicated neonatal hepatitis; jaundice that disappeared after comprehensive medical treatment for 2-4 weeks; and liver function that improved. The inclusion criteria for the congenital biliary atresia group were: biopsy results that suggested congenital biliary atresia; jaundice that did not subside after comprehensive medical treatment for 2-4 weeks; and liver function that did not improve. Eleven patients with congenital biliary atresia were confirmed after Kasai surgery or biliary angiography, and nine patients died during the 1- or 2-year follow-up.

\section{Immunohistochemical investigation}

Immunohistochemical staining was conducted by following the immunohistochemistry kit instructions, and light microscopy was used to evaluate the results. Anti-CTGF and anti-TGF- $\beta 1$ antibodies (mouse monoclonal antibodies) were both purchased from the R\&D Systems (Minneapolis, MN, USA), and immunohistochemistry kits were purchased from Dako (Glostrup, Denmark).

\section{Analysis}

TGF- $\beta 1$ and CTGF expression was compared with the blank negative control. The appearance of yellow or brown particles in the cytoplasm was taken as positive expression. Two blinded experienced pathologists randomly selected five horizons, calculated the average, and classified each sample into one of four levels according to range and intensity of staining as follows: negative (-), no staining; weakly positive (+), occasional positive staining of tissue, light yellow, up to $25 \%$; positive $(++)$, stained dark yellow, $26-50 \%$; positive $(+++)$, stained brown, more than $50 \%$.

\section{Statistical analysis}

All data are reported as means \pm SD (SPSS 11.5 statistical software package). The Fisher exact test, the $\chi^{2}$ test, and the $t$-test were used to compare groups. Level data were compared with 
the Mann-Whitney rank sum test, and correlation analysis was conducted by Spearman's rank correlation. $\mathrm{P}<0.05$ was considered statistically significant.

\section{RESULTS}

\section{General}

The 36 patients were classified into two groups based on the biopsy results and clinical prognoses. The neonatal hepatitis group comprised a total of 16 patients ( 9 males and 7 females). The congenital biliary atresia group comprised a total of 20 patients (12 males and 8 females). There was no significant difference in the gender ratio and no statistically significant differences in the average age, time of onset of jaundice, liver biopsy age, or direct or indirect bilirubin values between the two groups.

\section{Degree of hepatic fibrosis}

Fibrosis was graded by HE and Masson's trichrome staining. In the congenital biliary atresia group, there were $0,5,8,7$, and 0 patients with hepatic fibrosis in stages S0, S1, S2, S3, and $S 4$, respectively, while the corresponding numbers in the neonatal hepatitis group were 6,8 , 2,0 , and 0 patients, respectively. Hepatic fibrosis was more serious in the congenital biliary atresia group than in the neonatal hepatitis group, and the difference was significant $(P<0.01)$. There was no fibrosis in the two control patients.

\section{CTGF and TGF- $\beta 1$ expression in neonatal hepatitis and congenital biliary atresia patients}

In 3 of the 16 patients in the neonatal hepatitis group, CTGF was expressed in liver cells; in 2 cases, it was expressed in both liver cells and biliary epithelial cells. In 11 cases, TGF- $\beta 1$ was expressed in liver cells but not in biliary epithelial cells. In the congenital biliary atresia patients, CTGF and TGF- $\beta 1$ expression was observed in both liver cells and in biliary epithelial cells (Figures 1 and 2 ), and the differences were significant $(P<0.01)$. In the congenital biliary atresia patients, expression of CTGF and TGF- $\beta 1$ in liver tissue was significantly higher than in the neonatal hepatitis patients (Table 1$)$, and the differences were statistically significant $(P<0.01)$. No CTGF or TGF- $\beta 1$ expression was observed in the two control cases.

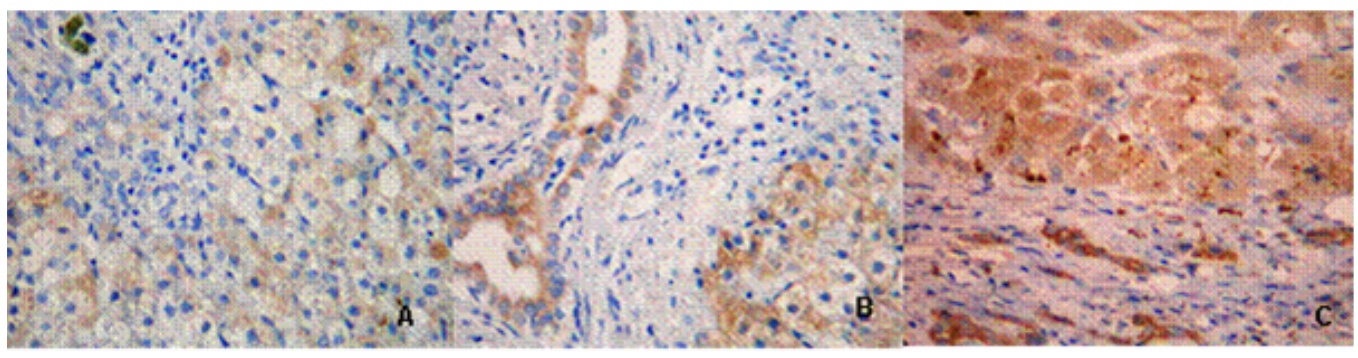

Figure 1. Transforming growth factor-b1 expression in congenital biliary atresia patients. Positive particles were visible in both bile duct epithelial cells and hepatocytes. A (+), B (++), C (+++) immunohistochemical method (200X). 


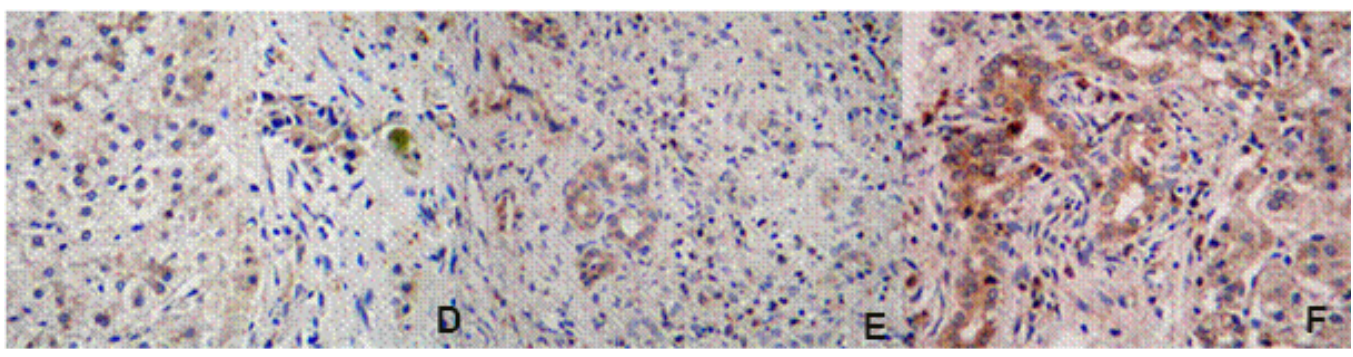

Figure 2. Connective tissue growth factor expression in congenital biliary atresia patients. Positive particles were visible in both bile duct epithelial cells and hepatocytes. D (+), E (++), F (+++) immunohistochemical method (200X).

Table 1. Transforming growth factor-b1 (TGF-b1) and connective tissue growth factor (CTGF) expression levels in neonatal hepatitis and congenital biliary atresia patients.

\begin{tabular}{l|c|c|c|c}
\hline \multirow{2}{*}{ Expression levels } & \multicolumn{2}{|c|}{ TGF- $\beta 1$} & \multicolumn{2}{c}{ CTGF } \\
\cline { 2 - 5 } & Neonatal hepatitis & Congenital biliary atresia & Neonatal hepatitis & Congenital biliary atresia \\
\hline- & 5 & 0 & 11 & 0 \\
\hline+ & 10 & 8 & 5 & 7 \\
\hline++ & 1 & 9 & 0 & 9 \\
\hline+++ & 0 & 3 & 0 & -4.734 \\
\hline$Z$-value & \multicolumn{2}{|c|}{-0.371} & & $<0.01$ \\
\hline$P$ value & $<0.01$ &
\end{tabular}

\section{CTGF and TGF- $\beta 1$ expression levels in the hepatocytes and biliary epithelial cells of the congenital biliary atresia patients}

CTGF and TGF- $\beta 1$ were expressed in both the hepatocytes and biliary epithelial cells of the congenital biliary atresia patients (Table 2), and the differences in expression levels in the biliary epithelial cells and liver cells were significantly different $(P<0.01)$.

Table 2. Transforming growth factor-b1 (TGF-b1) and connective tissue growth factor (CTGF) expression levels in liver cells and biliary epithelial cells of congenital biliary atresia patients.

\begin{tabular}{l|c|c|c|c}
\hline \multirow{2}{*}{ Expression levels } & \multicolumn{2}{|c|}{ TGF- $\beta 1$} & \multicolumn{2}{c}{ CTGF } \\
\cline { 2 - 5 } & Liver cells & Biliary epithelial cells & Liver cells & Biliary epithelial cells \\
\hline- & 0 & 0 & 0 & 0 \\
\hline+ & 12 & 2 & 10 & 2 \\
\hline++ & 6 & 15 & 9 & 14 \\
\hline+++ & 2 & 3 & 1 & 4 \\
\hline$Z$-value & \multicolumn{2}{|c|}{$<.291$} & \multicolumn{2}{c}{$<0.01$} \\
\hline P value & \multicolumn{2}{|c|}{$<01$} & & -3.497 \\
\hline
\end{tabular}

\section{Relationship between liver fibrosis and CTGF and TGF- $\beta 1$}

The Spearman's rank correlation analysis showed that, with aggravation of hepatic fibrosis, the CTGF and TGF- $\beta 1$ expression levels in the liver tissue gradually increased (Tables 3 and 4$)$, and the expression levels were significantly correlated $(P<0.01)$. 
Table 3. Liver fibrosis classification and connective tissue growth factor expression levels.

\begin{tabular}{l|c|c|c|c|c}
\hline Fibrosis classification & Number of cases & \multicolumn{3}{|c}{ Connective tissue growth factor expression levels } \\
\cline { 3 - 6 } & & $(-)$ & $(+)$ & $(++)$ & $(+++)$ \\
\hline $\mathrm{S}_{0}$ & 6 & 4 & 2 & 0 & 0 \\
\hline $\mathrm{S}_{1}$ & 13 & 6 & 6 & 1 & 0 \\
\hline $\mathrm{S}_{2}$ & 10 & 1 & 4 & 4 & 1 \\
\hline $\mathrm{S}_{3}$ & 7 & 0 & 0 & 4 & 3 \\
\hline Total & 36 & 11 & 12 & 9 & 4 \\
\hline
\end{tabular}

$R=0.741 ; P<0.01$

Table 4. Liver fibrosis classification and transforming growth factor- $\beta 1$ expression levels.

\begin{tabular}{l|c|c|c|c|c}
\hline \multirow{2}{*}{ Fibrosis classification } & Number of cases & \multicolumn{4}{|c}{ Transforming growth factor- $\beta 1$ expression levels } \\
\cline { 3 - 6 } & & $(-)$ & $(+)$ & $(++)$ & $(+++)$ \\
\hline$S_{0}$ & 6 & 2 & 4 & 0 & 0 \\
\hline$S_{1}$ & 13 & 2 & 8 & 3 & 0 \\
\hline$S_{2}$ & 10 & 1 & 5 & 4 & 0 \\
\hline$S_{3}$ & 7 & 0 & 1 & 3 & 3 \\
\hline Total & 36 & 5 & 19 & 10 & 3 \\
\hline
\end{tabular}

$R=0.584 ; P<0.01$.

\section{DISCUSSION}

The TGF- $\beta$ family of proteins comprises disulfide bond-linked basic polypeptides with a broad range of biological activities. There are five known TGF- $\beta$ forms: TGF- $\beta 1$, TGF- $\beta 2$, TGF- $\beta 3$, TGF- $\beta 4$, and TGF- $\beta 5$. TGF- $\beta 1$ has the highest activity in the liver and consists of two identical subunits that contain 112 amino acids. These two subunits form a dimer through a disulfide bond. TGF- $\beta 1$ has a wide potential impact on cell growth, differentiation, aggregation, and immune responses of the extracellular matrix. It is one of the most important liver fibrosis-inducing cytokines (Gressner et al., 2002; Miyazaki et al., 2005).

CTGF belongs to the CTGF/CYR61/NOV (CCN) family and can be produced by a variety of different cells. It is regulated by TGF- $\beta$, lipid peroxides, acetaldehyde, platelet-derived growth factor, tumor necrosis factor- $\alpha$, and other extracellular signals. Through autocrine and paracrine mechanisms, it regulates many cell functions, including growth, proliferation, apoptosis, adhesion, migration, differentiation, and extracellular matrix synthesis (Perbal, 2001). CTGF plays an important role in angiogenesis, cartilage formation, embryonic development, wound healing, tumor formation, organ fibrosis, and a variety of other biological processes (Rachfal and Brigstock, 2003).

Studies have shown that TGF- $\beta 1$ and CTGF play important roles in liver fibrosis. Using an RNase protection assay, Rachfal and Brigstock (2003) showed that, compared with the control group, in liver tissues from patients with primary biliary sclerosis, primary sclerosing cholangitis, or biliary atresia, CTGF mRNA increased by 3-5 times, and TGF- $\beta 1$ increased accordingly. AbouShady et al. (2000) used a northern blot to analyze the liver tissue of adults with chronic viral hepatitis, primary biliary sclerosis, primary sclerosing cholangitis, cryptogenic chronic liver disease, and alcoholic liver disease, and found that CTGF and TGF- $\beta 1$ expression levels were 6-8 times higher than in the normal population. In patients with chronic viral hepatitis, liver tissue CTGF protein expression level was associated with the fibrosis level. 
Congenital biliary atresia and neonatal hepatitis are the main causes of infant cholestasis. Early diagnosis and treatment have a great impact on prognosis. One of the most significant adverse prognostic factors is liver fibrosis. In a study of congenital biliary atresia patients, both TGF- $\beta 1$ and CTGF were found to increase. Ramm et al. (1998) found that TGF- $\beta 1$ mRNA expressed in biliary epithelial cells activated hepatic stellate cells (HSCs) and para-fibrosis cells in 18 congenital biliary atresia patients. Using an enzyme-linked immunosorbent assay, Tamatani et al. (1998) found that the level of serum CTGF in congenital biliary atresia patients was higher than in the control group, and was associated with the progression of liver fibrosis, suggesting that CTGF plays an important role in the development of the disease. Narkewicz et al. (2005) found that in congenital biliary atresia patients, CTGF expression levels in biliary epithelial cells and liver vascular endothelial cells were high, while in children with other liver diseases, CTGF expression was limited to HSCs and endothelial cells; in healthy children, no significant expression of CTGF was observed.

We found that CTGF and TGF- $\beta 1$ were expressed in hepatocytes and the cytoplasm of biliary epithelial cells in the positive expression samples of the two groups, but the expression levels were different. In the neonatal hepatitis group, the expression of CTGF and TGF- $\beta 1$ mainly occurred in the hepatocytes, and there was lower expression of these proteins in the biliary epithelial cells. In the congenital biliary atresia group, CTGF and TGF- $\beta 1$ were expressed in both hepatocytes and biliary epithelial cells, and the expression in biliary epithelial cells was stronger than in hepatocytes, which agreed with the results reported by Ramm et al. (1998) and Narkewicz et al. (2005). This suggests that, when biliary atresia occurs, biliary epithelial cell injury can act as a direct signal to start liver fibrosis. In the children with neonatal hepatitis, the expression in liver cells was high, which might have been because most of the children were still in the early stages of disease, and the liver fibrosis was less serious. However, this also indicated that the presence of liver cell inflammation can, to a certain extent, promote CTGF and TGF- $\beta 1$ expression, and may lead to the progression of liver fibrosis.

We also found that liver fibrosis occurred in both the neonatal hepatitis and congenital biliary atresia groups, and liver fibrosis was most serious in the congenital biliary atresia group. CTGF and TGF- $\beta 1$ expression levels in these two groups were different. Only 5 of the 16 patients with neonatal hepatitis had a CTGF expression level of $(+)$, and expression was negative in the other patients. In this group, there were 5 patients with a TGF- $\beta 1$ expression level of (-), 10 patients with an expression level of $(+)$, and 1 patient with an expression level of $(++)$. In the 20 patients with congenital biliary atresia, CTGF and TGF- $\beta 1$ expression levels were all $(+)$ to $(+++)$, further suggesting that liver fibrosis and CTGF/TGF- $\beta 1$ are closely related. Our results were similar to those reported by Kobayashi et al. (2005), who found that, after surgery, children with biliary atresia still had progressive liver fibrosis and portal hypertension, and CTGF levels were associated with the progression of liver fibrosis and poor prognosis.

In addition, with the aggravation of hepatic fibrosis, CTGF and TGF- $\beta 1$ expression levels in the liver tissue gradually increased. At the $S_{0}$ stage, there were few $(-)$ to $(+)$ results, and the expression levels were very low. With the increase in liver fibrosis, expression increased, and at the $\mathrm{S}_{3}$ stage, expression reached $(++)$ to $(+++)$, indicating that, with the increase of liver fibrosis, CTGF and TGF- $\beta 1$ expression levels also increased. Ding and Li (2006) also found that CTGF and TGF- $\beta 1$ expression increased with aggravation of hepatic fibrosis, but there was no significant correlation with inflammatory activity. The results were similar to those reported by Song et al. (2004) in rats with hepatic fibrosis. Considering that some postoperative biliary atresia patients and severe neonatal hepatitis patients experienced progressive liver fibrosis and, eventually, biliary cirrhosis, we believe that liver fibrosis is an important prognostic factor. CTGF and TGF- $\beta 1$ 
expression showed good correlation. Because TGF- $\beta 1$ is the most important factor in inducing collagen deposition and fibrosis, blocking the activation of latent TGF- $\beta 1$, inhibiting the interaction between ligand and receptor, and inhibiting downstream signal conduction are effective ways of treating fibrosis. However, because TGF- $\beta 1$ has antiproliferative and anti-inflammatory effects, longterm direct intervention of TGF- $\beta 1$ and its downstream molecules can have serious side effects. For example, the removal of the TGF- $\beta 1$ gene in mice led to death soon after birth owing to systemic inflammation caused by the loss of inhibition of the inflammatory process (Letterio and Böttinger, 1998). CTGF affects connective tissue cells and can maintain fibroblast phenotype. In the normal state, CTGF expression is very low. It is mainly expressed in stromal cells, and its role is also limited to the connective tissue. Thus, it is a more specific target, and blocking it may reverse fibrosis and herald a new era in the treatment of fibrotic diseases. George and Tsutsumi (2007) used CTGF siRNA in N-nitrosodimethylamine-induced mice liver fibrosis models, and discovered that CTGF and TGF- $\beta 1$ expression levels were significantly reduced, and connective tissue protein deposition in the liver was also reduced, suggesting that CTGF siRNA is a potential treatment for liver fibrosis.

In summary, we observed that liver fibrosis is present in both congenital biliary atresia and neonatal hepatitis patients, but liver fibrosis is more severe in congenital biliary atresia patients than in neonatal hepatitis patients. With the aggravation of hepatic fibrosis, CTGF and TGF- $\beta 1$ expression levels in liver tissue gradually increased, suggesting that CTGF and TGF- $\beta 1$ expression levels and liver fibrosis are closely related. In addition, CTGF and TGF- $\beta 1$ expression levels and tissue sources were different in congenital biliary atresia and neonatal hepatitis patients. In the former patients, expression was found in all sample biliary epithelial cells and hepatocytes, and the expression level was higher in biliary epithelial cells than in hepatocytes. In the latter patients, expression was limited to hepatocytes, and there was only slight expression in biliary epithelial cells. Considering the severity of liver fibrosis in congenital biliary atresia patients, early CTGF and TGF- $\beta 1$ expression in biliary epithelial cells may be involved in the pathogenesis of congenital biliary atresia.

\section{Conflicts of interest}

The authors declare no conflict of interest.

\section{ACKNOWLEDGMENTS}

Research supported by a grant from the Public Projects of Science Technology Department of Zhejiang Province (\#2014C33167).

\section{REFERENCES}

Abou-Shady M, Friess H, Zimmermann A, di Mola FF, et al. (2000). Connective tissue growth factor in human liver cirrhosis. Liver 20: 296-304. http://dx.doi.org/10.1034/j.1600-0676.2000.020004296.x

Ding $\mathrm{N}$ and $\mathrm{Li} \mathrm{Y}$ (2006). Transforming growth factor b1 and connective tissue growth factor in liver fibrosis. World Chin. J. Gastroenterol. 14: 2521-2526.

George J and Tsutsumi M (2007). siRNA-mediated knockdown of connective tissue growth factor prevents N-nitrosodimethylamine-induced hepatic fibrosis in rats. Gene Ther. 14: 790-803. http://dx.doi.org/10.1038/sj.gt.3302929

Gressner AM, Weiskirchen R, Breitkopf K and Dooley S (2002). Roles of TGF-beta in hepatic fibrosis. Front Biosci. 7: d793-d807. http://dx.doi.org/10.2741/gressner

Grotendorst GR, Lau LF and Perbal B (2000). CCN proteins are distinct from and should not be considered members of the insulin-like growth factor-binding protein superfamily. Endocrinology 141: 2254-2256. http://dx.doi.org/10.1210/ endo.141.6.7485 
Kasai M (1974). Treatment of biliary atresia with special reference to hepatic porto-enterostomy and its modifications. Prog. Pediatr. Surg. 6: 5-52.

Kobayashi H, Hayashi N, Hayashi K, Yamataka A, et al. (2005). Connective tissue growth factor and progressive fibrosis in biliary atresia. Pediatr. Surg. Int. 21: 12-16. http://dx.doi.org/10.1007/s00383-004-1254-z

Kurikawa N, Suga M, Kuroda S, Yamada K, et al. (2003). An angiotensin II type 1 receptor antagonist, olmesartan medoxomil, improves experimental liver fibrosis by suppression of proliferation and collagen synthesis in activated hepatic stellate cells. Br. J. Pharmacol. 139: 1085-1094. http://dx.doi.org/10.1038/sj.bjp.0705339

Lee H, Hirose S, Bratton B and Farmer D (2004). Initial experience with complex laparoscopic biliary surgery in children: biliary atresia and choledochal cyst. J. Pediatr. Surg. 39: 804-807. http://dx.doi.org/10.1016/j.jpedsurg.2004.02.018

Letterio JJ and Böttinger EP (1998). TGF-beta knockout and dominant-negative receptor transgenic mice. Miner Electrolyte Metab. 24: 161-167. http://dx.doi.org/10.1159/000057365

Lykavieris P, Chardot C, Sokhn M, Gauthier F, et al. (2005). Outcome in adulthood of biliary atresia: a study of 63 patients who survived for over 20 years with their native liver. Hepatology 41: 366-371. http://dx.doi.org/10.1002/hep.20547

Miyazaki T, Karube M, Matsuzaki Y, Ikegami T, et al. (2005). Taurine inhibits oxidative damage and prevents fibrosis in carbon tetrachloride-induced hepatic fibrosis. J. Hepatol. 43: 117-125. http://dx.doi.org/10.1016/j.jhep.2005.01.033

Narkewicz MR, Kasaragod A, Lucia MS, Pflummer S, et al. (2005). Connective tissue growth factor expression is increased in biliary epithelial cells in biliary atresia. J. Pediatr. Surg. 40: 1721-1725. http://dx.doi.org/10.1016/j.jpedsurg.2005.07.056

Nio M, Ohi R, Miyano T, Saeki M, et al. (2003). Five- and 10-year survival rates after surgery for biliary atresia: a report from the Japanese Biliary Atresia Registry. J. Pediatr. Surg. 38: 997-1000. http://dx.doi.org/10.1016/S0022-3468(03)00178-7

Perbal B (2001). NOV (nephroblastoma overexpressed) and the CCN family of genes: structural and functional issues. Mol. Pathol. 54: 57-79. http://dx.doi.org/10.1136/mp.54.2.57

Rachfal AW and Brigstock DR (2003). Connective tissue growth factor (CTGF/CCN2) in hepatic fibrosis. Hepatol. Res. 26: 1-9. http://dx.doi.org/10.1016/S1386-6346(03)00115-3

Ramm GA, Nair VG, Bridle KR, Shepherd RW, et al. (1998). Contribution of hepatic parenchymal and nonparenchymal cells to hepatic fibrogenesis in biliary atresia. Am. J. Pathol. 153: 527-535. http://dx.doi.org/10.1016/S0002-9440(10)65595-2

Song SL, Gong ZJ and Zhang QR (2004). Expression and significance of TGF- $\beta 1$, Smad3, Smad7, CTGF expression in experimental hepatic fibrosis rats. Shaanxi J. Med. 33: 486-490.

Tamatani T, Kobayashi H, Tezuka K, Sakamoto S, et al. (1998). Establishment of the enzyme-linked immunosorbent assay for connective tissue growth factor (CTGF) and its detection in the sera of biliary atresia. Biochem. Biophys. Res. Commun. 251: 748-752. http://dx.doi.org/10.1006/bbrc.1998.9543 